\title{
Achromatic Four Quadrant Phase Mask using the Dispersion of Form Birefringence
}

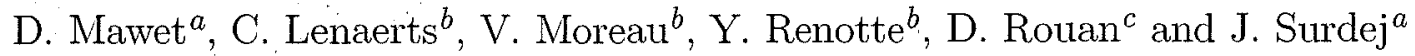 \\ ${ }^{a}$ IAGL, Université de Liège, 17,B5C Allée du 6 Août, B-4000 Sart Tilman, Belgium \\ ${ }^{b}$ HOLOLAB, Université de Liège, 17,B5 Allée du 6 Août, B-4000 Sart Tilman, Belgium \\ ${ }^{c}$ DESPA, Observatoire de Paris-Meudon, 5 pl J. Janssen, F-92195 Meudon, France
}

\begin{abstract}
We describe an alternative design for the 4-quadrant phase mask coronagraph described recently by Rouan et al. $2000 .^{1}$ Based on the same principle, i.e. producing a very efficient nulling by mutually destructive interferences of the coherent light from the main source, our mask realises the $\pi$ phase shift using some properties of ZOGs (Zeroth Order Gratings) and according to an original scheme respecting the 4-quadrant symmetry. When the period of the one-dimension grating structure is smaller than the wavelength of the incident light, the structure becomes birefringent. The effective refractive indices depend on the wavelength. Using this feature, we can design a mask whose nulling efficiency is maintained within a wide wavelength range. Numerical simulations were made according to the RCWT (Rigorous Coupled Wave Theory).
\end{abstract}

Keywords: Coronagraphy, 4-quadrant phase mask, achromaticity, subwavelength gratings, ZOGs, form birefringence

\section{INTRODUCTION}

The 4-quadrant phase mask (FQ-PM) divides the focal plane in 4 equal areas centered on the optical axis, with 2 of them providing a $\pi$ phase shift (see Fig. 1). This causes destructive interference to occur inside the geometric pupil area. Then, the traditional Lyot stop is required to block the rejected starlight. In the perfect case, one can show that the rejection of this design is infinite. But in practice, several parameters limit the

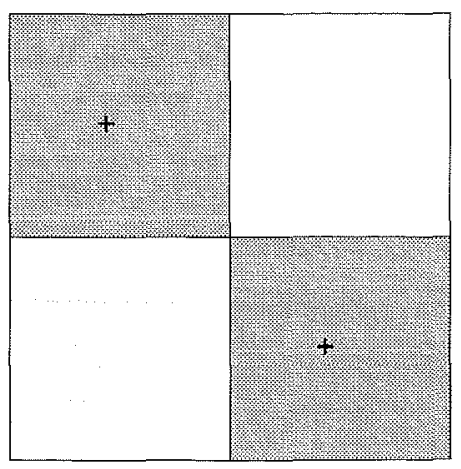

Figure 1. 4-quadrant phase mask scheme.

dynamical range of this phase coronagraph: aberrations of the optical train, departure of the pupil shape from centro-symetry, phase shift chromaticity,... (see Riaud et al. 2001²)

Our aim is to provide an original solution to the problem of the phase shift chromaticity. Indeed, for evident reasons of detectability, one has to increase the spectral bandwidth but without degrading the rejection ratio

Further author information: (Send correspondence to D. Mawet)

D. Mawet: E-mail: Dimitri.Mawet@student.ulg.ac.be 
$R=\frac{I_{\text {with } F Q-P M}}{I_{\text {without } F Q-P M}}$. The latter is dependent on the phase shift quality (variance) $\sigma^{2}$ over the chosen wavelength range, by the relation $R=\frac{2}{\sigma^{2}}$. To implement this achromatic phase shift, some solutions have been proposed up to now, from thin film stacks to dispersive bulk material approaches, in reflection and in transmission (see Riaud et al. $2001^{2}$ ).

The principal motivation for the "grating approach" comes from the fact that, quite apart from their optical performances, gratings are monolithic or quasi-monolithic and introduce therefore few foreign materials. This situation is very unlike the case of multilayer thin films which lead to problems associated with limitations in the coating materials (IR) and various physical and chemical properties that affect adhesion, thermal mismatch, stability and durability of the thin-film stack. Moreover, due to the geometry of the FQ-PM, such decaying physico-chemical interactions could occur at the joints of quadrants, even for bulk material approaches. These effects are amplified in the infrared because of the various cryogenic thermic constraints.

\section{ZOGS}

ZOGs (Zeroth Order Gratings) consist in general of subwavelength gratings, i.e. the period of the structure is smaller than the wavelength of the incident light. They do NOT diffract light in the sense that only zeroth transmitted and reflected orders are allowed to propagate outside the grating regions, leaving incident wave fronts free from any further aberrations.

Indeed, whether a diffraction order propagates or not is determined by the well-known grating equation from which we can thus find a "ZOG condition" on the grating period to wavelength ratio $\frac{\Lambda_{x}}{\lambda}$ :

$$
\frac{\Lambda_{x}}{\lambda}<\frac{1}{\max \left(n_{i}, n_{t}\right)+n_{i} \sin \theta}
$$

with $\theta$, the incidence angle, $n_{i}$ and $n_{t}$, the refractive indices of the incident and transmitting media, respectively.

To simulate grating responses in the subwavelength and resonant domains, scalar theories fail dramatically. The vectorial nature of light must be taken into account...

\section{DISPERSION OF FORM BIREFRINGENCE}

Interaction between the periodically modulated permittivity and the vectorial electromagnetic field inside a grating leads to interesting effects on the phases and amplitudes of the external propagating fields.

For example, one dimension ZOGs turn out to be artificially birefringent, that's what is called the form birefringence $\Delta n_{\text {form }}=n_{\text {eff }}^{T E}-n_{\text {eff }}^{T M T}$. In the quasi-static limit, i.e. when the ratio $\frac{\Lambda_{x}}{\lambda}<<1$, simple homogeneization treatment involving average considerations leads to the two following effective indices corresponding to TE (Transverse Electric) and TM (Transverse Magnetic) states of polarisation (see Born \& Wolf ${ }^{3}$ ):

$$
\begin{aligned}
& n_{e f f, 0}^{T E}=\left(F \epsilon_{a}+(1-F) \epsilon_{b}\right)^{\frac{1}{2}} \\
& n_{e f f, 0}^{T M I}=\left(\frac{\epsilon_{a} \epsilon_{b}}{F \epsilon_{b}+(1-F) \epsilon_{a}}\right)^{\frac{1}{2}}
\end{aligned}
$$

where $\left(\epsilon_{a}\right)^{\frac{1}{2}}=n_{a}$ and $\left(\epsilon_{b}\right)^{\frac{1}{2}}=n_{b}$ are the structure's real indices, and where $\mathrm{F}$ is the fill factor (ratio of the width of grating grooves to the period, $b / \Lambda_{x}$ ). This immediate approach is called the Zero ${ }^{\text {th }}$ Order Effective Medium Theory (EMT0).

But when the ratio $\frac{\Lambda_{x}}{\lambda}$ is no longer negligible, the latter closed-form expressions for the effective refractive indices are no longer correct. In such a case, second order EMT (EMT2) which is deduced from the electromagnetic propagation in stratified media theory, allows to derive these expressions for the effective indices (see Rytov $\left.1956^{4}\right)$ :

$$
n_{e f f, 2}^{T E}=\left(n_{e f f, 0}^{T E}+\frac{1}{3}\left(\frac{\Lambda}{\lambda}\right)^{2} \pi^{2} F^{2}(1-F)^{2}\left(n_{a}^{2}-n_{a}^{2}\right)^{2}\right)^{\left(\frac{1}{2}\right)}
$$




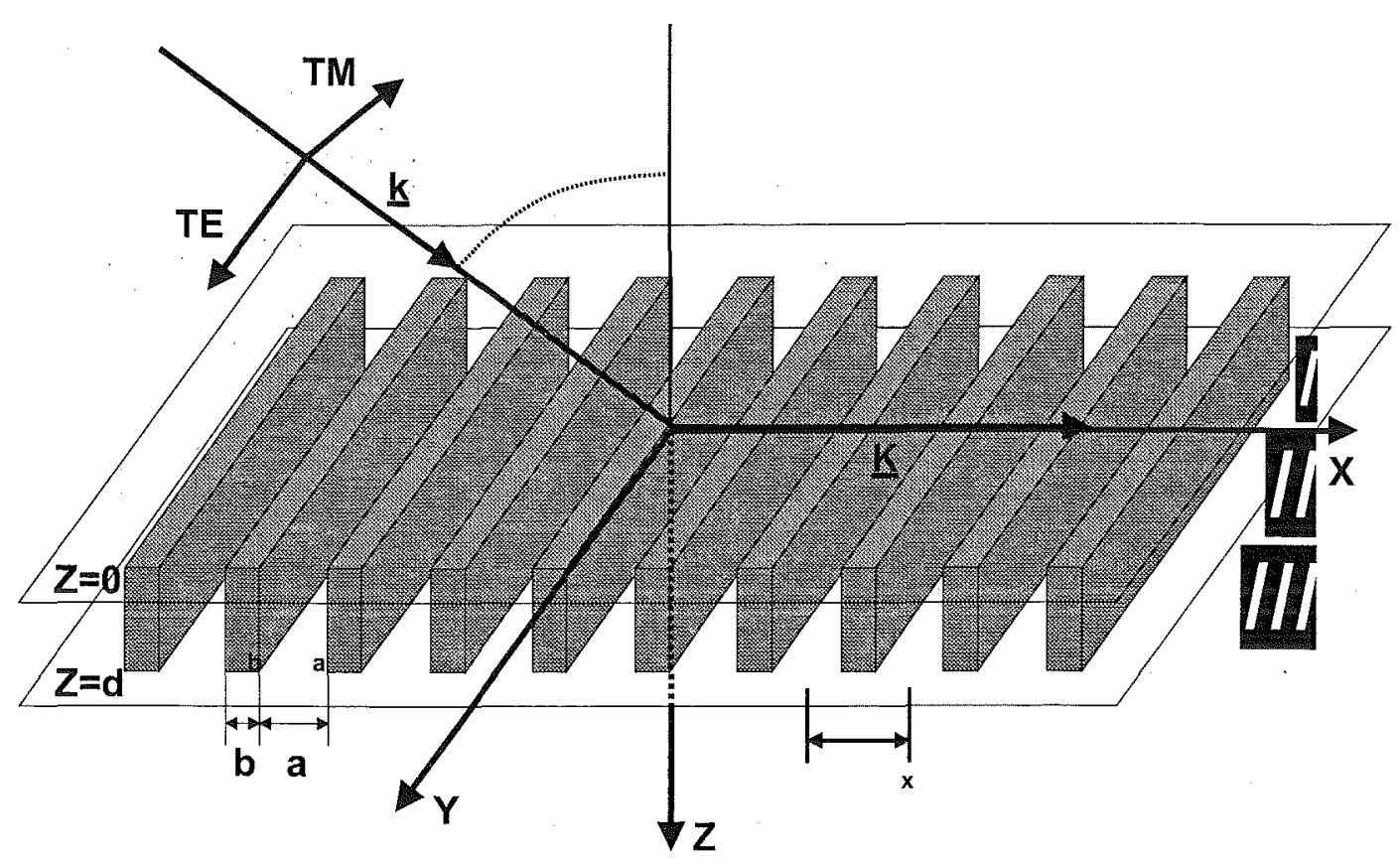

Figure 2. Surface relief grating. Geometry of the diffraction problem. Parameter definitions. Vectorial decomposition of incident natural light.

$$
n_{e f f, 2}^{T M I}=\left(n_{e f f, 0}^{T M I}+\frac{1}{3}\left(\frac{\Lambda}{\lambda}\right)^{2} \pi^{2} F^{2}(1-F)^{2}\left(\frac{1}{n_{a}^{2}}-\frac{1}{n_{a}^{2}}\right)^{2}\left(n_{e f f, 0}^{T M}\right)^{6}\left(n_{e f f, 0}^{T E}\right)^{2}\right)^{\left(\frac{1}{2}\right)}
$$

In addition to the dependence on the ratio $\frac{\Lambda_{x}}{\lambda}$, thus on the wavelength, we can also notice the dependence of the effective indices versus other parameters available in a design procedure: the grating period $\Lambda_{x}$, the fill factor $\mathrm{F}$, the grating depth $h$ and the grating real indices $n_{a}$ and $n_{b}$.

The wavelength dependence of the effective indices is consequently also found in the form birefringence $\Delta n_{\text {form }}$. This phenomenon is called the dispersion of form birefringence. We must emphasize the term "form". Indeed, this property is essentially given by the geometry, no longer by the intrinsic characteristics of the materials.

\section{IDEAL BIREFRINGENCE}

The propagation in a birefringent medium along the optical axes leads to the following phase shift between ordinary and extraordinary wave components:

$$
\Delta \phi=\frac{2 \pi}{\lambda} \Delta n l
$$

where $\Delta n$ is the birefringence and $l$, the propagation path length. One searches the ideal birefringence which could compensate for the hyperbola dependence of the phase shift expression (6) so as to catch an achromatic phase shift. The latter, in the case of a $\pi$ phase shift should be:

$$
\Delta n_{\text {form }}=\frac{\lambda}{2 h}
$$

By carefully selecting the grating parameters (incidence should also be considered), it is possible to make the form birefringence tangent to the ideal birefringence (see Fig. 3), thus partially compensating for the $\frac{1}{\lambda}$ dependence of the phase shift expression within an appreciable wavelength range (see Kikuta et al. $1997^{5}$ ). 


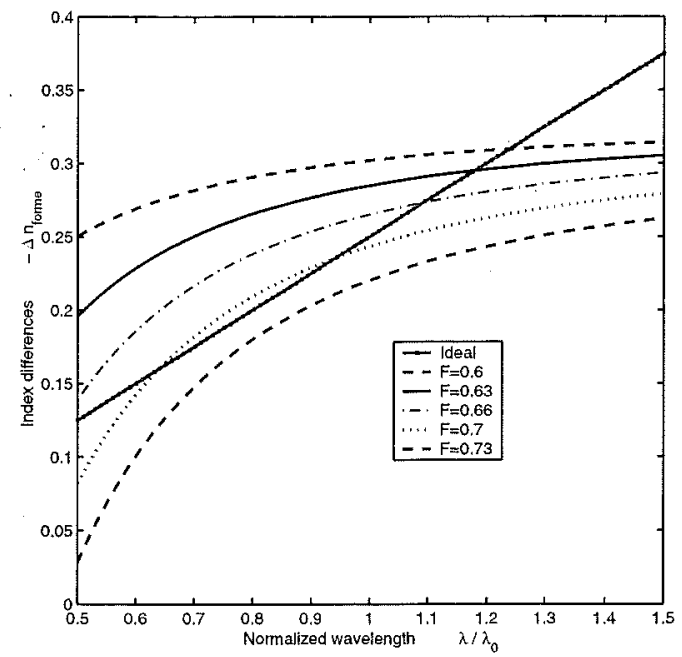

Figure 3. Search of tangence between linear ideal birefingence and effective ones.

\section{SIMULATIONS AND DESIGN}

Effective Medium Theories deal only with zeroth order diffracted waves and disregard the evanescent waves, which are higher-order diffracted waves. To obtain the exact response of the design, we must perform calculations based on the Rigorous Coupled Wave Theory (RCWT) (see for example Moharam \& Gaylord 19826). Moreover, this theory does take into account external media influences in terms of multiple-beam interferences and gives in addition to the phase, the intensity of the diffracted waves (diffraction efficiencies).

\subsection{Spectral Domain}

We have chosen the $\mathrm{K}$ band $(2-2.5 \mu \mathrm{m})$ to optimise the achromatic phase shifter, for two main reasons:

- first, this wavelength range corresponds to the optimal band of the NAOS adaptative module of the VLT which is an interesting ground-based candidate for the FQ-PM implementation;

- finally, the $\mathrm{K}$ band is one of the best compromise between contrast and resolution for the observation of many science objects of modern coronagraphy (search for exo-zodi, exo-planets,...).

\subsection{Material Choice}

The most appropriate dielectric in this context is Silicon:

- for its high index;

- for its low and known dispersion (including Temperature dependence) *;

- for its transparency in the considered infrared domain (K Band);

- for its widespread utilisation in electronics (easy manufacturing). 

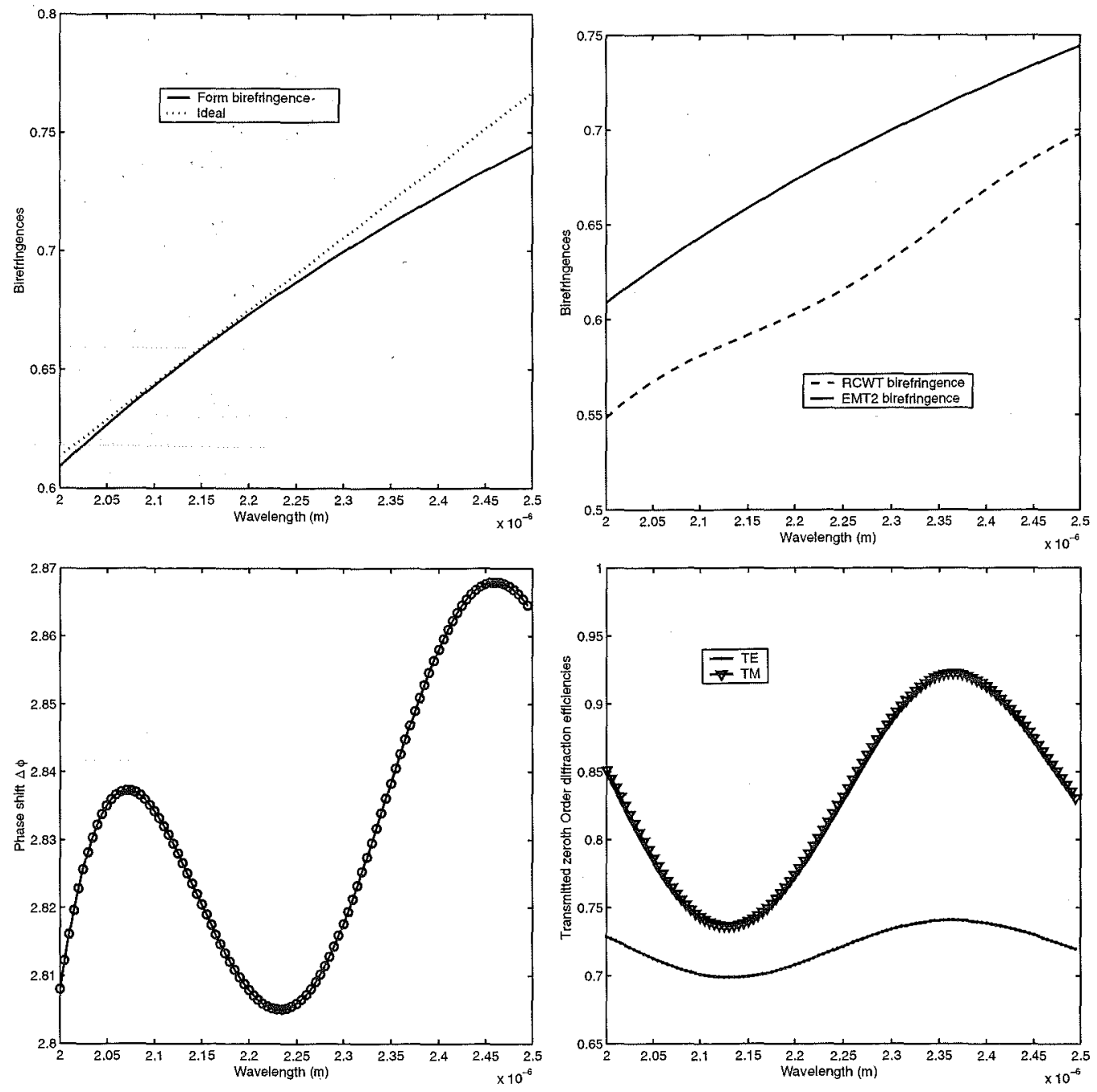

Figure 4. Top-left: tangency between the effective form birefringence and the ideal one. Top-right: effective form birefringence calculated with EMT2 and RCWT. Bottom-left: phase shift of the simple lamellar grating designed by EMT2 but calculated with RCWT. Bottom-right: diffraction efficiency of the transmitted zeroth-order for the TE and TM states of polarisation calculated with RCWT.

\subsection{Simple lamellar grating}

In the academic diffraction problem resolution, three media are taken into account: the incident medium, the modulated region and the transmitting medium. The first idea is to consider the following configuration: Air/Siair/Si, i.e. the incident medium is air, the modulated region is a surface relief grating composed of air and $\mathrm{Si}$ imprinted in a Silicon substrate which is the transmitting medium. Our aim is thus to make the effective form birefringence tangent to the ideal one. To do this, one can play with the grating parameters available. Doing so, we get at the tangence, the following set of grating parameters, calculated in a first step, for rapidity and facility considerations, by EMT2:

${ }^{*}$ Sellmeier's dispersion relation for Silicon: $n^{2}(T, \lambda)=\epsilon+\frac{A}{\lambda^{2}}+\frac{B \lambda_{1}^{2}}{\left(\lambda^{2}-\lambda_{1}^{2}\right)}$, with $\lambda_{1}=1.1071 \mu m, \epsilon=1.16858 \times 10^{1}$, $A=9.39816 \times 10^{-1}$ and $B=8.10461 \times 10^{-3}$ 


\begin{tabular}{|c|c|}
\hline$\Lambda_{x}$ & $443 \mathrm{~nm}$ \\
\hline $\mathrm{h}$ & $1.63 \mu \mathrm{m}$ \\
\hline $\mathrm{F}$ & 0.87 \\
\hline
\end{tabular}

But we have seen above that these results are in fact not accurate enough and incomplete. Using the RCWT, we can see in figure 4 (top-right) that the actual birefringence was overestimated by the EMT2. Moreover, if we get interested in the diffraction efficiencies in transmission, we remark that they are very different and much too low (see Fig. 4).

\subsection{Sandwich structure}

The reason of such a bad result for the transmittances is that the difference of the effective indices that leads to the birefringence is also responsible for the index differences resulting in unequal Fresnel reflections at the interfaces between the modulated region and the external ones. One way to reduce these unwanted reflections is to consider the following sandwich structure (see Kikuta et al. $1997^{5}$ ): Si/Si-air/Si.

Indeed, while doing so we reduce index differences at the first interface. Here are the new optimized parameters:

\begin{tabular}{|c|c|}
\hline$\Lambda_{x}$ & $427 \mathrm{~nm}$ \\
\hline $\mathrm{h}$ & $1.56 \mu \mathrm{m}$ \\
\hline $\mathrm{F}$ & 0.83 \\
\hline
\end{tabular}

and the following design responses in terms of phase shift quality and transmittances (see Fig. 5):
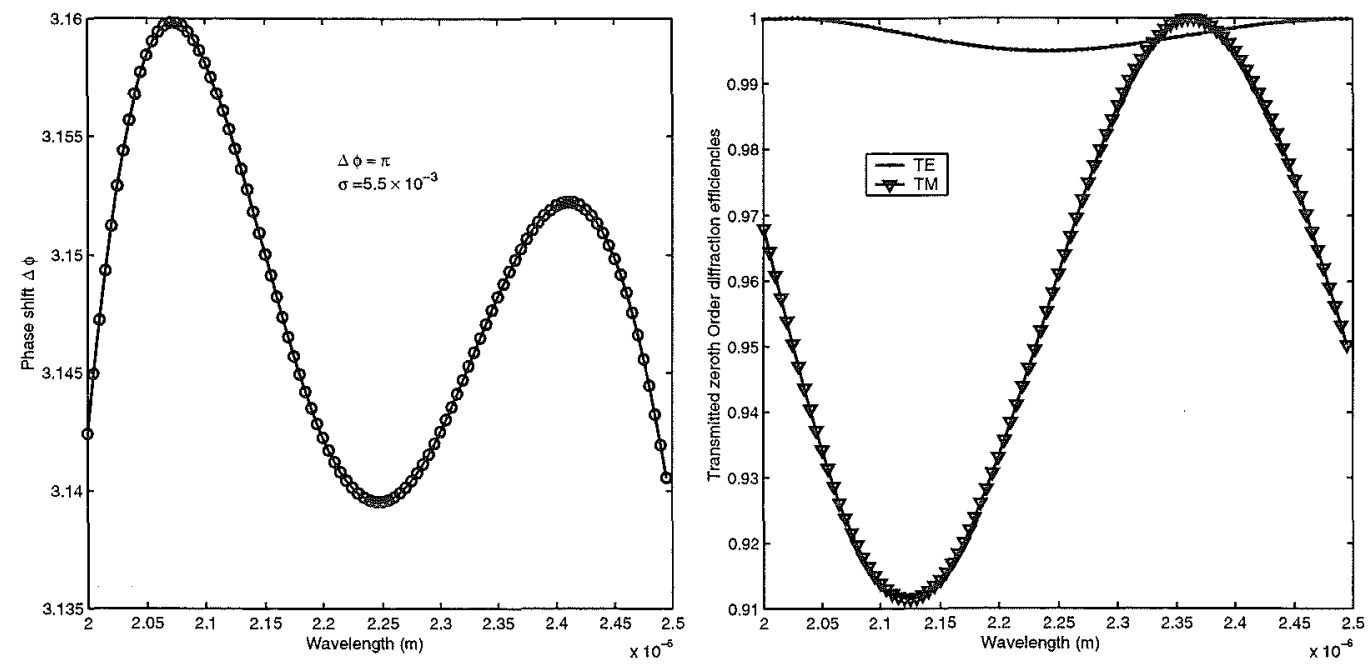

Figure 5. Left: optimized phase shift for the sandwich structure. Right: optimized transmittance for the sandwich structure.

The calculated phase shift quality is $\sigma=5.5 \times 10^{-3} R M S$ which leads to a rejection ratio $R=55000$ if the transmittances were perfectly equal. This is not the case but the results are quite good with a transmittance superior to $91 \%$ for TM and superior to $99 \%$ for TE. 


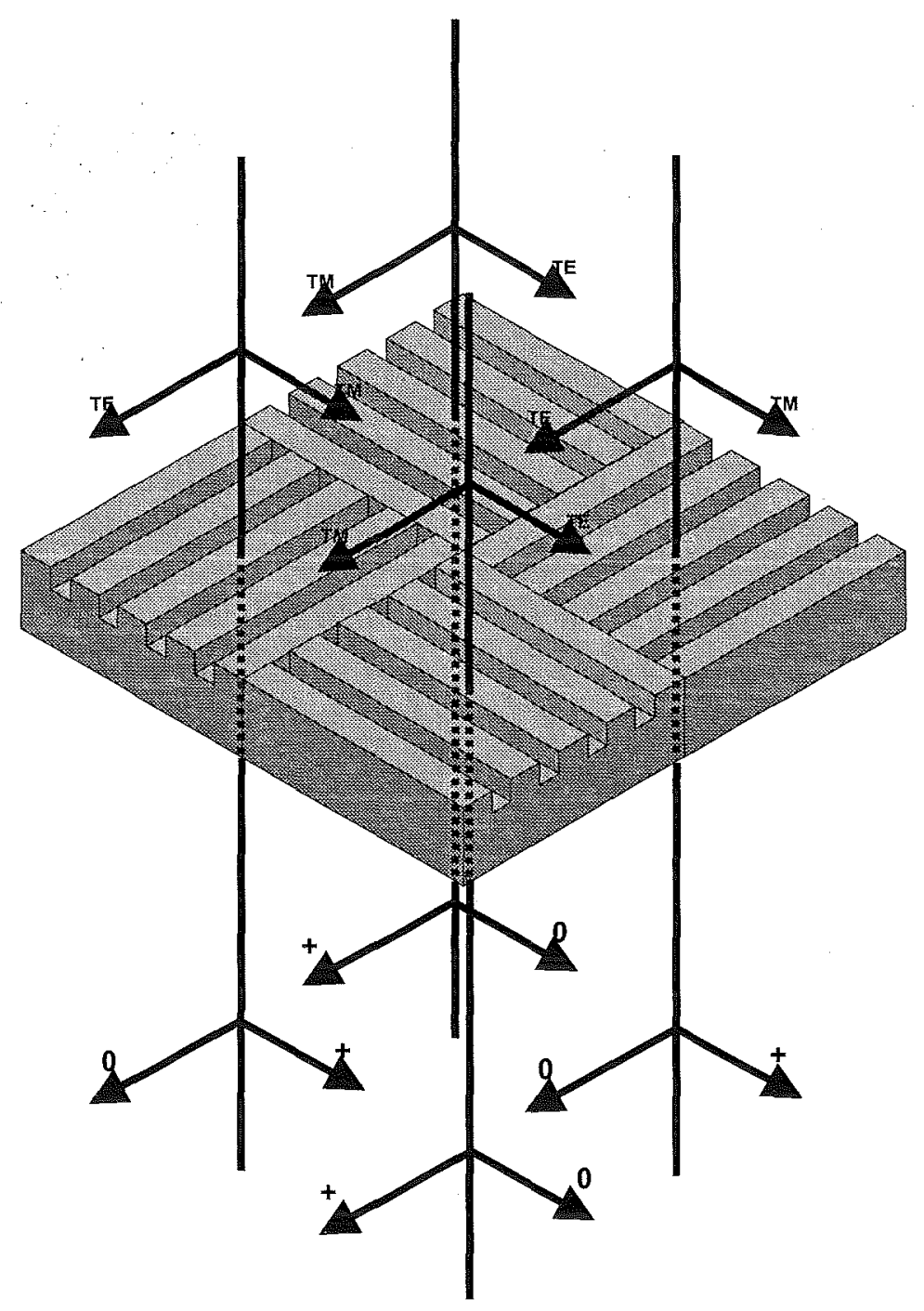

Figure 6. Implementation of the gratings on the FQ-PM.

\section{IMPLEMENTATION AND MANUFACTURING}

\subsection{Implementation on the FQ-PM}

Implementation of this differential phase shift in nulling systems as phase coronagraphs is quite immediate and elegant. Considering two identical gratings rotated by 90 degrees from one another, we retrieve for each state of polarisation the "designed" achromatic phase shift (see Fig. 6). So it works with natural light. As we have the same component (under the hypothesis of perfect replication) just rotated in each quadrant of the coronagraph (see Fig. 6), the only resultant phase shift is the designed differential one.

\subsection{Tolerances of the Design and Manufacturing}

One can show that the achromaticity of the design can be more easily controlled than the exact and essential $\pi$ phase shift. However, the flexibility of the design procedure allows to compensate for the lack of precision of a single parameter by variation of other ones, including a posteriori possibilities with the incidence.

Thus taking the latter remark into account, systematic variations of the grating parameters lead to the following tolerances of the design in terms of phase shift quality conservation: 
- the fill factor must be controlled within $1 \%$, i.e. $\approx 5 \mathrm{~nm}$;

- the etch depth within $\approx 10 \mathrm{~nm}$;

- the normal incidence within $\approx 10$ degrees;

- the temperature within $\approx 40 \mathrm{~K}$.

These specifications on the grating parameter precisions are within the most advanced nanotechnology techniques available in microelectronics industry, such as Electron-Beam lithography and Reactive Ion Etching, their tight control is nevertheless critical and requires systems equipped with in situ monitoring possibilities (see Lalanne et al. $1999^{7}$ ).

\section{LIMITATIONS AND SOLUTIONS}

Quite apart from manufacturing constraints, there are several intrinsic limitations of the current design. The first limiting characteristic is the difference in transmission for the TM and TE directions of polarization that will be responsible for incomplete nulling. The second comes from the fact that the lower the variance $\sigma^{2}$, the better the rejection.

Several families of solutions do exist:

- exploiting material combination possibilities;

- playing with the grating profile (triangular, cosinusoidal, trapezoidal...);

- using conical incidence (the plane of incidence does not contain the grating vector);

- multiple gratings approach;

- implementing phase subtraction techniques.

These families are detailed in the following paragraphs.

\subsection{Material Combination Possibilities}

By choosing an appropriate material combination, the differences between grating effective indices and external region real ones can be strongly reduced and equalized.

To minimize index differences, the best material to choose for outside regions should be equally spaced between the two effective indices associated with the modulated region. In the case of our sandwich structure, the effective indices are in average equal to 3.2 for TE polarisation and 2.4 for TM. So the ideal refractive index for the external region should be approximatively 2.8. This is the case for CdTe.

Indeed, the hypothetical structure CdTe/SU8-Si/CdTe gives transmittances superior to $97 \%$ for $\mathrm{TE}$ and superior to $99 \%$ for TM. The SU8 is a low index organic resist. Its purpose is double:

- planarize the surface relief grating;

- reduce the real index difference of the modulated region in order to reduce the TM effective index dispersion for better control of the unwanted reflections over the whole bandwidth.

More details and relevant technological indications can be found in the literature (see, for example, Deguzman $\&$ Nordin $2001^{8}$ ). 


\subsection{Best Grating Profile Quest}

Varying the grating profile gives a possibility to extend the wavelength band and phase shift quality. For example, triangle profiles present the best $1 \mathrm{D}$ AR behaviour but a small dispersion, not sufficient to compensate for the hyperbola dependence of the phase shift expression. An attractive compromise is the trapezoidal profile, situated between triangle and rectangular ones, which combines both their AR and dispersion properties (see Richter et al. $\left.1995^{9}\right)$.

\subsection{Incidence}

Taking into account conical incidence (where the plane of incidence does not contain the grating vector $\mathrm{K}$ ) allows to relax the "ZOG threshold period". Increasing the grating period increases birefringence and thus reduces etching depth. Furthermore, achromatic retarders have already been designed considering only incidence variation instead of fill factor (see Bokor et al. $2001^{10}$ ). To our knowledge, no simulation has yet been undertaken using at the same time the fill factor and incidence angle variations. It could be a fruitful way to experiment both technique advantages.

Incidence variation could also be considered to implement afterwards design response corrections. Indeed, non conical incidence variation already induces, beyond tolerance values, translation of phase shift profile (almost conserving global achromaticity).

\subsection{Double Grating}

Another way to relax manufacturing constraints is to divide the phase shift process. Doing so, it can be shown that the overall design response is also optimized. Let us consider the double grating configuration shown in figure 7. If the substrate depth is superior to the coherence length of the incoming light ${ }^{\dagger}$, the two gratings can be considered independently so that the total phase shift is the sum of the two individual ones. The

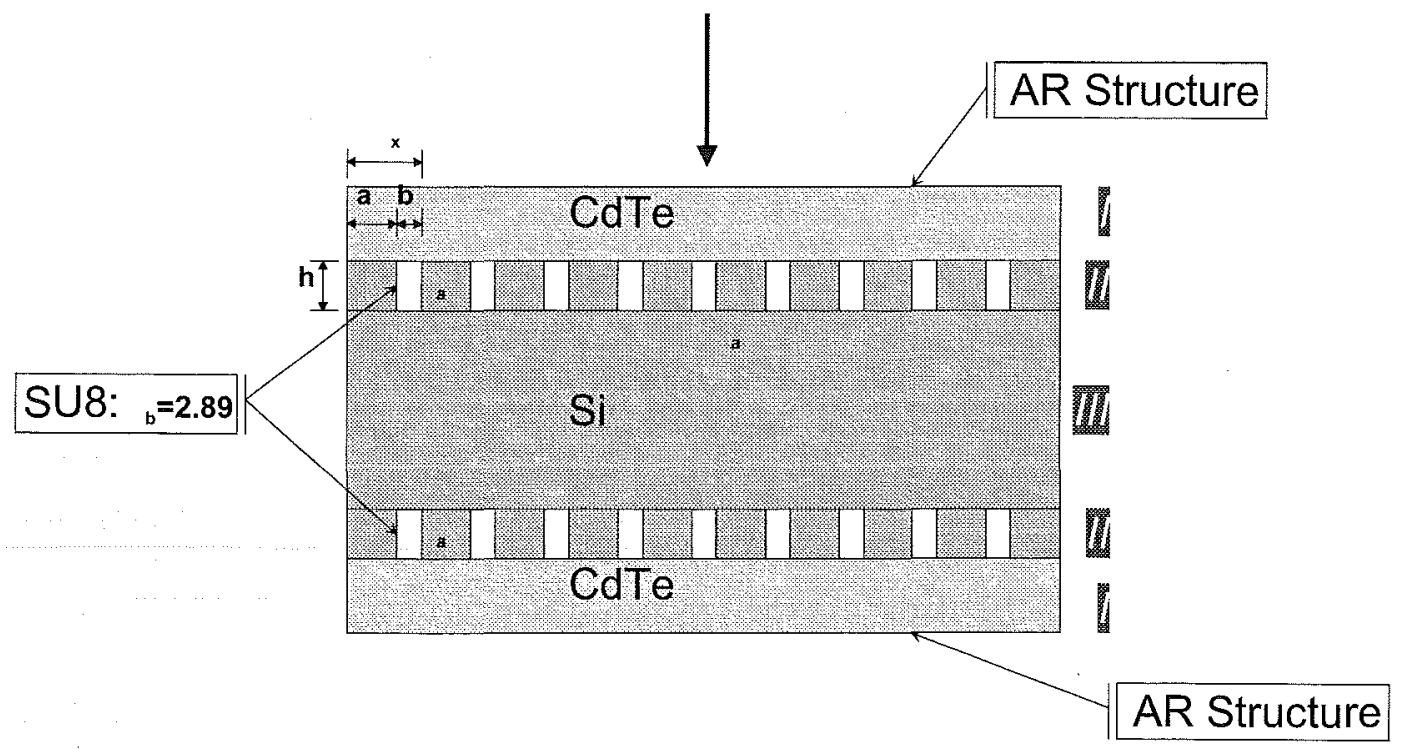

Figure 7. Double grating phase shifter.

results obtained are encouraging: $\sigma=3.2 \times 10^{-3}$ RMS for the first configuration (double Si/air-Si/Si) and $\sigma=6.6 \times 10^{-3} R M S$ for the second (double CdTe/SU8-Si/Si). For the latter, the gain in the transmittance equality is appreciable with more than $99 \%$ for both TM and TE.

Here are the corresponding grating parameters for the $\pi / 2$ phase shifters:

\footnotetext{
${ }^{\dagger}$ With a $\mathrm{K}$ filter, $\approx 10 \mu \mathrm{m}$ so a physical depth in Silicon of $\approx 3 \mu \mathrm{m}$
} 


\begin{tabular}{|c|c|c|}
\hline Configuration & Si/air-Si/Si & CdTe/SU8-Si/Si \\
\hline$\Lambda_{x}$ & $494 n m$ & $542 \mathrm{~nm}$ \\
\hline $\mathrm{h}$ & $680 \mathrm{~nm}$ & $1.81 \mu \mathrm{m}$ \\
\hline $\mathrm{F}$ & 0.7 & 0.77 \\
\hline
\end{tabular}

where we can appreciate the fabrication constraints relaxing especially in the etching depth.

\subsection{Phase Subtraction}

This well-known technique (fòr example, see Yip et al. $1996^{11}$ ), based on dispersion compensations, has to be reconsidered. Indeed, advantage can be taken from the fact that the latter can be controlled (form birefringence!).

\section{CONCLUSIONS}

We have proposed an alternative design for the achromatic 4-quadrant phase mask using some properties of ZOGs with encouraging results. Indeed, the optimized gratings show very good achromatic performances over the whole $\mathrm{K}$ band and several differential transmittance control possibilities.

As a conclusion, we point out the flexibility of the entire approach. This flexibility is based on the number of free parameters available in the design procedure. Moreover, given the speed at which microelectronic technologies are currently developing, more accurate control of more and more parameters will be possible every year, opening the door to rapid substantial performance improvement.

\section{REFERENCES}

1. D. Rouan, P. Riaud, A. Boccaletti, Y. Clnet, and A. Labeyrie, "The four-quadrant phase-mask coronagraph. i. principle," PASP 112, p. 1479, 2000.

2. P. Riaud, A. Boccaletti, D. Rouan, F. Lemarquis, and A. Labeyrie, "The four-quadrant phase-mask coronagraph. ii. simulations," PASP 113, p. 1145, 2001.

3. Born and Wolf, Principles of Optics, ch. 15.5.2 Form birefringence. Cambridge University Press, 7 ed., 1999.

4. S. Rytov, "Electromagnetic properties of a finely stratified medium," JETP Soviet Physics 2, p. 466, 1956.

5. H. Kikuta, Y. Ohira, and K. Iwata, "Achromatic quarter-wave plates using the dispersion of form birefringence," Applied Optics 36(7), p. 1566, 1997.

6. M. Moharam and T. Gaylord, "Diffraction analysis of dielectric surface-relief gratings," JOSA 72, p. 1385, 1982.

7. P. Lalanne, P. Pichon, P. Chavel, E. Cambril, and H. Launois, "Interferometric characterization of subwavelength lamellar gratings," Applied Optics 38(23), p. 4980, 1999.

8. P. Deguzman and G. Nordin, "Stacked subwavelength gratings as circular polarization filters," Applied Optics 40, p. 5731, 2001.

9. I. Richter, P.-C. Sun, F. Xu, and Y. Fainman, "Design considerations of form birefringent microstructures," Applied Optics 34(14), p. 2421, 1995.

10. N. Bokor, R. Shechter, N. Davidson, A. A. Friesem, and E. Hasman, "Achromatic phase retarder by slanted illumination of a dielectric grating with period comparable with the wavelength," Applied Optics 40(13), p. 2076, 2001.

11. W. Yip, H. Huang, and H. Kwok, "Achromatic wave retarder by phase subtraction," Applied Optics 35(22), p. $4381,1996$. 